\title{
Reappearing Primary User Detection in FBMC/OQAM Cognitive Radios
}

\author{
Tero Ihalainen, Ari Viholainen, Tobias Hidalgo Stitz, and Markku Renfors \\ Department of Communications Engineering, Tampere University of Technology \\ P.O. Box 553, FI-33101 Tampere, Finland, Email: tero.j.ihalainen@tut.fi
}

\begin{abstract}
This paper investigates the problem of detecting the reappearance of a primary user signal during ongoing secondary data transmission. The high frequency containment of filter bank multicarrier (FBMC) waveforms is exploited to construct, in spectrally efficient manner, continuous silent subbands within the transmission band for spectrum monitoring. Energy detection is carried out on these monitoring subbands in secondary receivers. The distribution of the test statistic is derived to characterize the detector performance in the transient phase where the reappearance of the primary signal falls arbitrarily within the integration window. The experimental data obtained from an FBMC/OQAM secondary multiplexing testbed verifies the derived analytic model. Further, the impact of the transmit power amplifier nonlinearity on the proposed spectrum monitoring scheme is discussed.
\end{abstract}

\section{INTRODUCTION}

Over the last decade, wireless communication markets have seen both ever-growing interest to broadband wireless applications and services on one hand, and emergence of new wireless devices with accelerating pace on the other. These trends drastically fuel the demand for radio frequency spectrum. However, the conventional spectrum regulatory framework, where each operator is granted an exclusive license in a certain frequency band, fails to respond to this increased demand of spectrum. Indeed, spectrum usage measurements reveal that the licensed spectral resources are severely underutilized both in time and over space. Clearly, there exists a striking contradiction between the spectrum scarcity inflicted on the emerging wireless services and the low utilization of the assigned resources in the licensed bands due to inflexible static frequency allocations.

To resolve this paradox, a new spectrum usage strategy called dynamic spectrum access (DSA) [1] has been proposed. In such a scheme, unlicensed (secondary) users are allowed to opportunistically utilize the temporally and/or spatially unused licensed (primary) bands, referred to as spectrum holes or white spaces, provided that the legacy system can operate free from secondary interference.

Cognitive radio (CR) [2], [3] technology is seen as a key enabler of DSA. CR is a smart secondary user (SU) device, which senses and monitors the radio frequency environment in its operating range to analyze the primary user (PU) spectrum occupancy and to look for spectrum holes with the aim of reconfiguring its transmission accordingly. Therefore, two key functionalities are required from a CR device: i) spectrum sensing (SS) and ii) waveform adaptation.

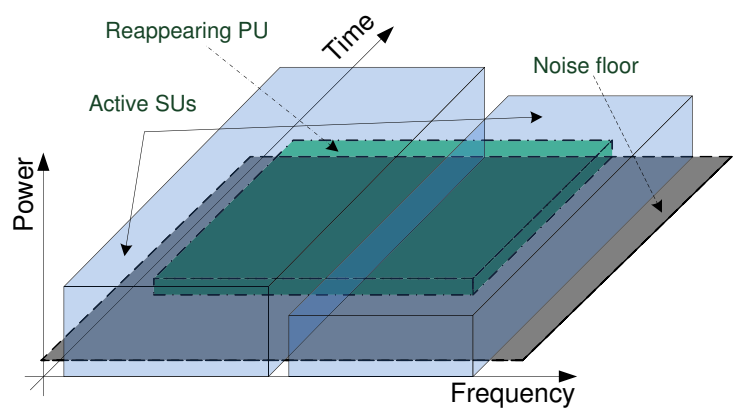

Fig. 1. Spectrum monitoring: the challenge of detecting a reappearing PU during ongoing secondary communication.

For SS, a number of approaches have been investigated in the literature, which can coarsely be categorized into matched filter detection, energy detection, and cyclostationary feature detection techniques, each with their individual pros and cons [4]. Approaches differ from each other in terms of implementation complexity, amount of prior knowledge required, processing gain obtained, and detection time required. Waveform adaptability, in terms of center frequency, bandwidth, and power etc., is required to respond to the dynamically changing operation environment. Multicarrier modulation techniques are strong candidates for the required spectrally-agile physical layer solution. This is due to the inherent ability to perform spectrum sensing on one hand and the flexibility of resource allocation on the other.

The majority of the existing SS literature focuses on the pre-transmission sensing, which is targeted for spectrum hole acquisition. However, to appropriately protect the PUs, also the ability to efficiently and robustly detect the presence of a reappeared PU signal, in parallel to ongoing secondary communication, is of crucial importance. This mode of PU sensing, referred hereafter to as spectrum monitoring and illustrated in Fig. 1, is necessary to guarantee fast reaction and channel vacation to minimize the interference inflicted on the legacy system. A common approach for spectrum monitoring is to perform periodic SS [5] where secondary transmission is switched off every once in a while to create a silent sensing interval for probing the channel.

In recent $\mathrm{CR}$ literature, filter bank based signal processing has been recognized as a high performance toolbox particularly well-suited for the required $\mathrm{CR}$ transceiver processing, both from the SS [3], [6] and secondary data multiplexing point of views [7], [8]. In this paper, we follow this line of re- 


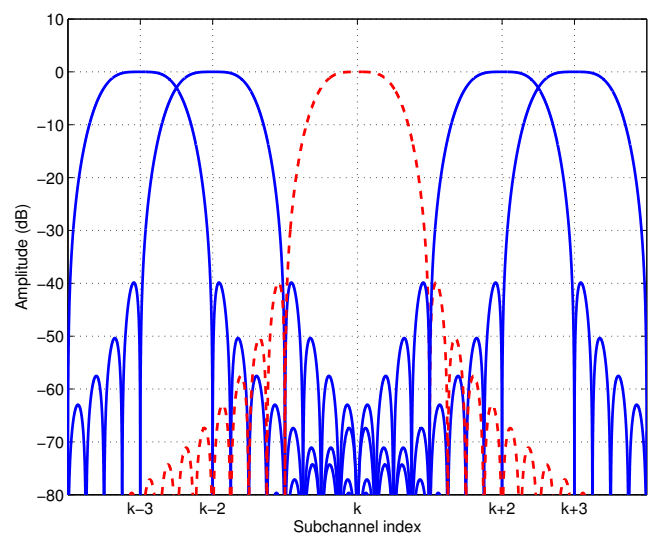

Fig. 2. Subcarrier frequency responses in FBMC with an NPR prototype filter of [10]. Overlapping factor $K=4$. Solid lines: active secondary subcarriers. Dashed line: a monitoring subcarrier.

search and assume a secondary system based on the filter bank multicarrier (FBMC) technique in combination with offset QAM (OQAM) subcarrier modulation [9]. In what follows, the spectrum monitoring problem in data-receiving FBMC/OQAM secondary receivers is investigated. Specifically, a concept of silent sensing subbands, stemming from a particularity of the FBMC approach, is presented.

The rest of this paper is organized as follows. Section II explains the principles of the FBMC monitoring technique. In Section III, an analytical model for the transient phase energy detector performance is derived, including an analysis on the impact of the sensing filter response. The effect of transmit power amplifier nonlinearity on the proposed scheme is discussed in Section IV. Section V presents the results of a numerical performance evaluation.

\section{SPECTRUM MONITORING IN FBMC RECEIVERS}

Opposite to periodic sensing intervals, the scheme proposed here is based on the idea of leaving narrow parts of the spectrum unused in secondary transmission, allowing them to be utilized for spectrum monitoring in a continuous manner. The feasibility to do this, in spectrally efficient manner, is fundamentally grounded on the impressive frequency containment characteristic of the FBMC transmission schemes. Indeed, in FBMC, with properly designed prototype filter, one subcarrier is sufficient to isolate a sensing subcarrier from active datacarrying subcarriers of the adjacent secondary systems. Figure 2 demonstrates this in case of a near perfect reconstruction (NPR) prototype filter designed with the frequency sampling technique [10].

For a detailed description of the overall transmultiplexer structure and the signal model in FBMC/OQAM communications, a reader is guided to [8], [9] and the references therein. Here, the focus is on specific receiver baseband functions to facilitate the forthcoming analysis of the sensing subband scheme. Figure 3 shows the main baseband processing elements of an FBMC/OQAM receiver, specifically, the analysis filter bank (AFB), which implements demodulation, and the following subchannel processing. In an FBMC/OQAM re-

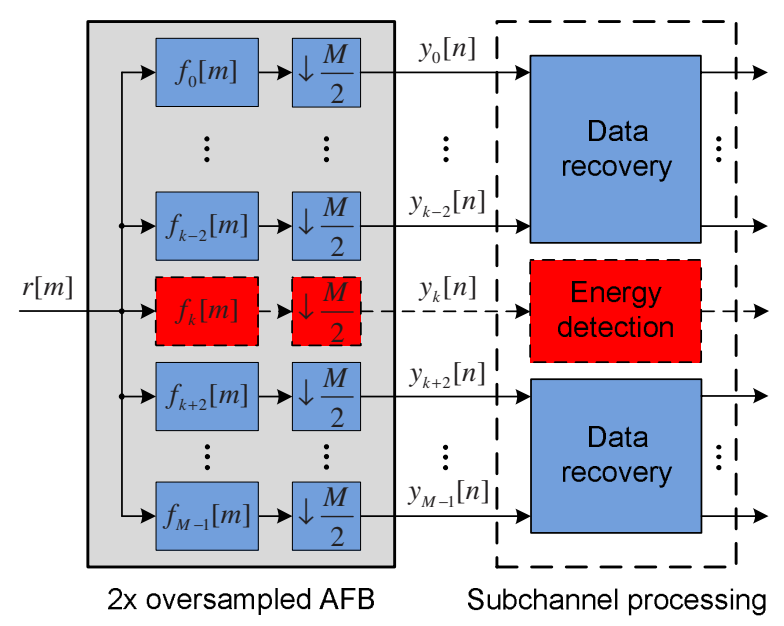

Fig. 3. A baseband processing structure for simultaneous FBMC/OQAM data reception and spectrum monitoring.

ceiver, subchannel processing, e.g., fractionally-spaced equalization and frequency domain fine synchronization are carried out at $2 \mathrm{x}$ oversampled rate. In other words, complex-valued subchannel samples are processed instead of the real-valued ones, which are finally sufficient for data reception at the end subchannel processing chain. The 2x oversampled OQAMsubsymbol rate sequence at the input of the subchannel processing block can be expressed as

$$
y_{l}[n]=\left[r[m] \star f_{l}[m]\right]_{\downarrow \frac{M}{2}}
$$

where $r[m], \star, f_{l}[m]$, and $\downarrow \frac{M}{2}$ denote the high-rate signal at the input of the AFB, the convolution, the lth analysis filter, and the downsampling by $\frac{M}{2}$, respectively. The sequence $y_{k}[n]$, at the output of the sensing subchannel filter $(l=k)$, can be utilized for spectrum monitoring. In this paper, this sequence of samples is considered to be used for energy detection, as discussed in more details in the following section.

\section{ENERGY DETECTION IN MONITORING SUBBANDS}

We consider spectrum monitoring in an FBMC/OQAM receiver, where energy detection is carried out at subchannel level at the output of the analysis bank. In energy detection, the test statistic is obtained as

$$
T(\mathbf{Y})=\frac{1}{N} \sum_{n=0}^{N-1}|Y[n]|^{2}
$$

where $Y[n], n=0,1, \ldots, N-1$, are complex independent (uncorrelated) observations. The binary hypothesis testing is carried out based on the test statistic. Using Gaussian approximation, the distributions in the absence (hypothesis $\mathcal{H}_{0}$ ) and presence (hypothesis $\mathcal{H}_{1}$ ) of the PU signal can be written as [11]

$$
\begin{aligned}
&\left.T(\mathbf{Y})\right|_{\mathcal{H}_{0}} \sim \mathcal{N}\left(\sigma^{2}, \frac{1}{N} \sigma^{4}\right) \\
&\left.T(\mathbf{Y})\right|_{\mathcal{H}_{1}} \sim \mathcal{N}\left(P+\sigma^{2}, \frac{1}{N}\left(P+\sigma^{2}\right)^{2}\right),
\end{aligned}
$$

respectively. Here, $P$ and $\sigma^{2}$ denote the signal and noise variances, respectively. 


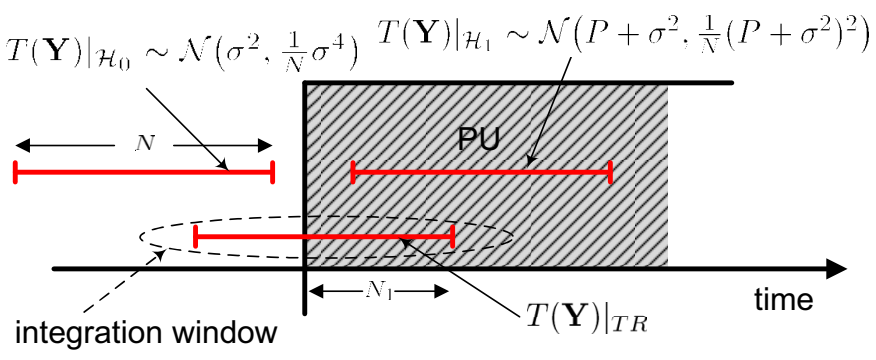

Fig. 4. A model for the distribution analysis of the transient phase test statistic.

\section{A. Reappearing PU and transient phase detection perfor- mance}

Typically, it is assumed that the PU is either absent or active during the whole sensing interval used for constructing the test statistic. In practice, it may happen that the PU becomes active (reappearing $P U$ ) during the measurement period. In such cases, only some fraction of the integration window, say $0<N_{1}<N$ samples, are measured after PU becomes active. Such a transient phase and the related test statistic distributions are illustrated in Fig. 4.

The distribution of the transient phase test statistic, $\left.T(\mathbf{Y})\right|_{T R}$, can be derived as follows. The integration window can virtually be split into two distinct sub-windows such that one of them contains only those observation samples before PU becomes active $\left(N-N_{1}\right.$ samples; see Fig. 4) while the other one contains the rest $N_{1}$ samples. The distributions corresponding to the sample subsets within these virtual sub-windows write $\mathcal{N}\left(\sigma^{2}, \frac{1}{N-N_{1}} \sigma^{4}\right)$ and $\mathcal{N}\left(P+\sigma^{2}, \frac{1}{N_{1}}\left(P+\sigma^{2}\right)^{2}\right)$, respectively. The overall sequence of $N$ samples can be interpreted as a linear combination of these independent normal random variables using relative weights of $\frac{N-N_{1}}{N}$ and $\frac{N_{1}}{N}$, respectively. Next, by making use of the following property of the normal distribution: given

$$
\begin{aligned}
& \left\{\begin{array}{l}
X_{1} \sim \mathcal{N}\left(\mu_{1}, \sigma_{1}^{2}\right) \\
X_{2} \sim \mathcal{N}\left(\mu_{2}, \sigma_{2}^{2}\right)
\end{array}\right. \\
& \Rightarrow a X_{1}+b X_{2} \sim \mathcal{N}\left(a \mu_{1}+b \mu_{2}, a^{2} \sigma_{1}^{2}+b^{2} \sigma_{2}^{2}\right)
\end{aligned}
$$

we obtain an expression for the mixture-distribution as

$\left.T(\mathbf{Y})\right|_{T R} \sim \mathcal{N}\left(\sigma^{2}+\frac{N_{1}}{N} P, \frac{1}{N}\left(\left(1-\frac{N_{1}}{N}\right) \sigma^{4}+\frac{N_{1}}{N}\left(P+\sigma^{2}\right)^{2}\right)\right)$.

The probability of detecting the presence of a PU signal during the transient phase can be expressed as

$$
P_{D, T R}=\operatorname{Pr}\left(\left.T(\mathbf{Y})\right|_{T R}>\gamma\right),
$$

i.e., as the tail probability towards $+\infty$ over the distribution in (5), with respect to a given decision threshold $\gamma$. The probability $P_{D, T R}$ can be observed to gradually increase within the lower and upper limits of $\operatorname{Pr}\left(\left.T(\mathbf{Y})\right|_{\mathcal{H}_{0}}>\gamma\right)=P_{F A}$ and $\operatorname{Pr}\left(\left.T(\mathbf{Y})\right|_{\mathcal{H}_{1}}>\gamma\right)=P_{D}$, respectively.

\section{B. Impact of the sensing filter response}

Recall that in FBMC, subcarrier signal samples are obtained at a rate, which is two times the subcarrier bandwidth. The samples are correlated, but there remains the question whether there is some benefit from using all the samples instead of the ones at symbol rate. The effect of the sensing filter frequency response is analyzed in general form in [12]. Assuming that the sensing filter frequency response is $F(j \omega)$ and it is normalized in such a way that $\frac{T}{2 \pi} \int|F(j \omega)|^{2} d \omega=1$, the means of both test statistics of (3) remain the same but the variances of both test statistics are multiplied by the factor

$$
\beta=\frac{T}{2 \pi} \int|F(j \omega)|^{4} d \omega .
$$

In FBMC context, $F(j \omega)$ is the prototype filter frequency response. With the considered prototype filter design [10], the impulse response coefficients of the prototype filter can be expressed using a closed-form representation as

$$
p[m]=\frac{1}{K M}\left(\bar{P}[0]+2 \sum_{k=1}^{K-1}(-1)^{k} \bar{P}[k] \cos \left(\frac{2 \pi k}{K M}(m+1)\right)\right),
$$

where $K=4, m=0,1, \ldots, K M-2, \bar{P}[0]=1$, $\bar{P}[1]=0.97195983, \bar{P}[2]=1 / \sqrt{2}$, and $\bar{P}[3]=\sqrt{1-\bar{P}[1]}=$ 0.23514695 . For this particular filter $\beta=0.8228$. The variance of the test statistic is reduced by this factor, under both hypotheses, and consequently the needed sample complexity is reduced by the same factor in subcarrier-wise sensing. On the other hand, when symbol-rate samples are used, aliasing results in effectively constant sensing filter frequency response over the subchannel bandwidth, and $\beta=0.5$. In summary, $N$ samples at subcarrier symbol rate and $2 \beta N=1.65 N$ samples at $2 \mathrm{x}$ oversampled rate result in the same $P_{F A}$ and $P_{M D}$. The needed time record length is reduced by the factor of 0.8228 in the latter case.

\section{EFFECT OF THE POWER AMPLIFIER NONLINEARITY}

Figure 5 shows a subchannel-level signal model for an FBMC secondary device, which performs sensing subband based spectrum monitoring. The depicted exemplary case shows a dedicated sensing subband isolated by singlesubchannel guard bands from the surrounding active secondary signal multiplexes SU X and SU Y. The secondary user signalto-noise ratio (SU SNR) defines the received SU signal power relative to the noise floor in the secondary receiver. Similarly, the nominal primary user signal-to-noise ratio (PU SNR) characterizes the level of the received PU signal power (under $\mathcal{H}_{1}$ ) in comparison to the noise floor. The PU SNR and SU SNR(s), jointly with the SU transmitter power amplifier (PA) characteristics, set the requirements for the energy detector. Running the PA in its nonlinear operation region results in outof-band spectral power leakage, which in turn will increase the effective level of the noise experienced by the energy detector in the spectrum monitoring subcarriers at the SU receiver. 


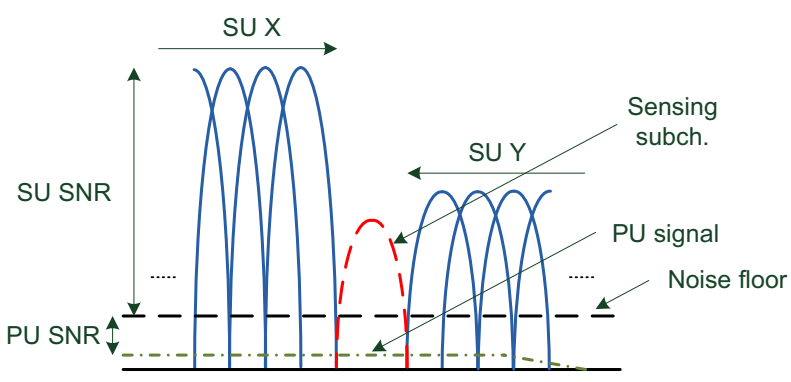

Fig. 5. Signal model for the sensing subchannel based spectrum monitoring analysis.

The effective PU SNR can be expressed as $P / \bar{\sigma}^{2}$, where the effective noise variance is given by

$$
\bar{\sigma}^{2}=\sigma^{2}+\sum_{j=1}^{2} \cdot 10^{S N R_{d B}^{j} / 10} \cdot \sigma^{2} \cdot 10^{\alpha_{d B}^{j} / 10} .
$$

Here, $S N R_{d B}^{j}$ and $\alpha_{d B}^{j}$ denote the power of the SU $j$ (located next to the monitoring subcarrier in frequency) w.r.t. the noise floor and the PA back-off dependent relative (w.r.t. to the SU signal power) level of the spectral regrowth component, respectively.

\section{NUMERICAL PERFORMANCE EVALUATION}

The performance of the continuous sensing subband based monitoring scheme has been tested through simulations. The proposed monitoring scheme was analyzed by running energy detection at a dedicated sensing subband in an FBMC/OQAM secondary receiver, during active secondary data multiplexing, according to the signal model of Fig. 5. The secondary transmission was modeled using following system parameters: sampling frequency of $1.4 \mathrm{MHz}$, transform size $M=128$, an NPR prototype filter of (8), and assuming AWGN channel. The PU signal was modelled as a wideband (with respect to the bandwidth of the sensing subband) single-carrier signal with raised cosine pulse shaping. Moreover, in all the experiments considered, the sensing subband was located within the bandwidth occupied by the reappearing primary signal. In the analysis, we considered a realistic PA model ${ }^{1}$, which is specified in details in [13] and for which relative power levels of the spectral regrowth component, corresponding to different PA input back-off (IBO) values, are tabulated in Table I.

At first, we investigate how accurately the performance of the silent subband detector in the experimental testbed reflects that of the analytical model in (6). Figures 6 and 7 show the transient phase detection probability, $P_{D, T R}$, as a function of $N_{a}$, a parameter equivalent to $N_{1}$ at the 2 x oversampled rate (i.e., a number of fractionally-spaced post-reappearance samples in the integration window). Figures 6 and 7 show the detection probability in cases of PU SNR $=-6 \mathrm{~dB}$ with $N_{f}=336$ samples and PU SNR $=-10 \mathrm{~dB}$ with $N_{f}=2016$ samples, respectively, where $N_{f}$ denotes the integration period

\footnotetext{
${ }^{1}$ The analytic baseband model has been derived by measuring a Class $\mathrm{A}$ $\mathrm{GaAs}$ InGaP HBT power amplifier. The AM/AM and AM/PM characteristics of the PA model were defined by fitting the parameters of the analytic SSPA and TWT models [14], respectively, based on the measurement data.
}

TABLE I

TABLE I. SPECTRAL REGROWTH CHARACTERISTICS OF THE CONSIDERED PA MODEL. TOP ROW: INPUT BACK-OFF $(d B)$. BOTTOM ROW: RELATIVE POWER LEVEL $(d B)$ TWO SUBCARRIER SPACINGS FROM THE ACTIVE BAND EDGE SUBCARRIER $\left(\alpha_{d B}\right.$ IN (9)).

\begin{tabular}{|c|c|c|c|c|c|c|}
\hline \hline 5.5 & 6.0 & 7.5 & 9.0 & 11.0 & 12.0 & 18.0 \\
\hline-25.0 & -26.7 & -31.0 & -35.5 & -42.0 & -45.1 & -56.9 \\
\hline
\end{tabular}

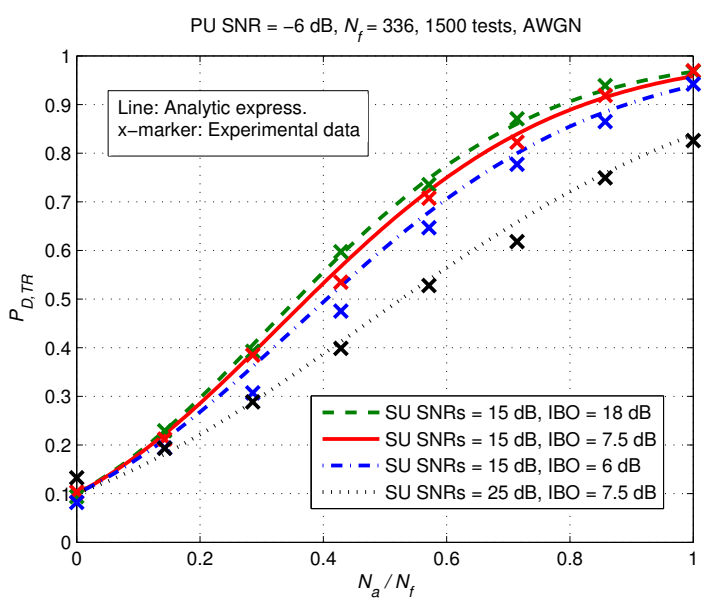

Fig. 6. Comparison between the analytic and experimental transient phase detection performances. The case with PU SNR $=-6 \mathrm{~dB}$ and $N_{f}=336$.

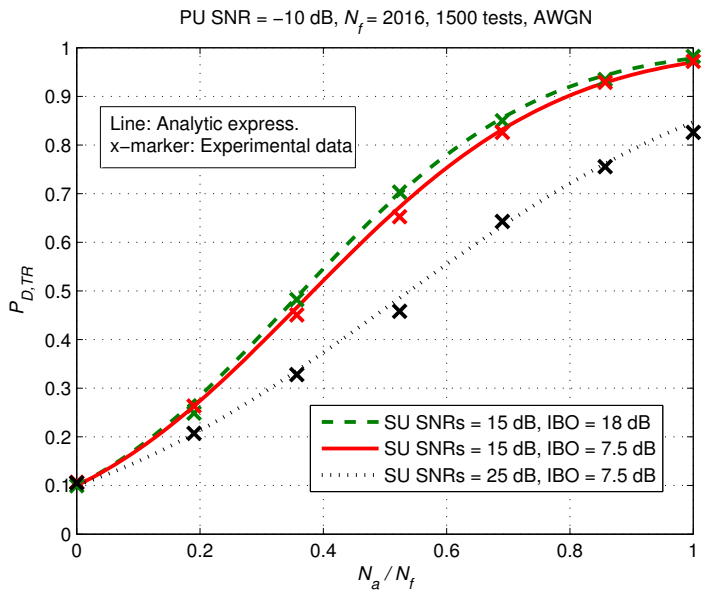

Fig. 7. Comparison between the analytic and experimental transient phase detection performances. The case with PU SNR $=-10 \mathrm{~dB}$ and $N_{f}=2016$.

in fractionally-spaced samples $\left(N_{f}=2 N\right)$. The simulated results are based on 1500 tests. The decision threshold $\gamma$ is designed to achieve a target false alarm probability of $P_{F A}=0.1$ with $N_{a}=0$. The effective noise variance $\bar{\sigma}^{2}$ is assumed to be perfectly known. The experimental performance can be seen to match fairly accurately with that of the analytical model for different levels of signal band power dynamics (SU SNRs) and PA nonlinearity (SU IBOs).

Figures 8 and 9 show the analytic transient phase detection probability as a function of the nominal PU SNR. A fixed-size integration window with $N_{f}=300$ samples is used for detection. Performance is shown for a number of alternative cases corresponding to different PU reappearance times compared to 


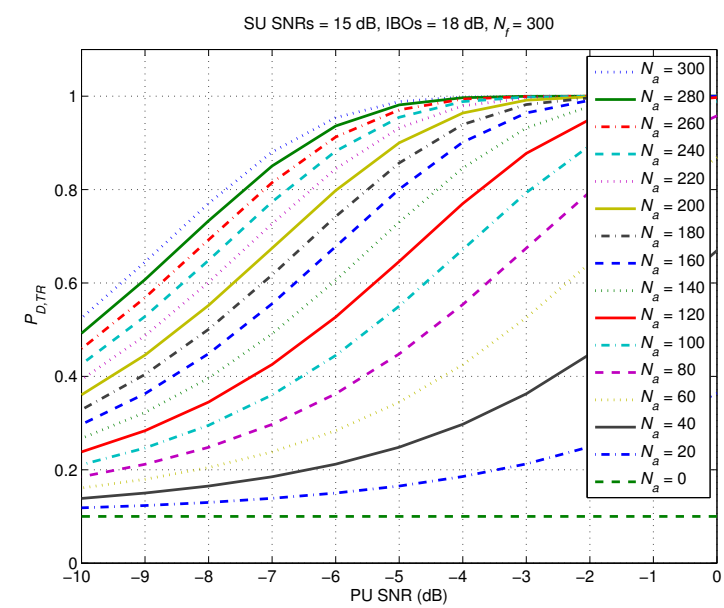

Fig. 8. Transient phase detection performance. The case with modest signal band power dynamics and linear PA operation.

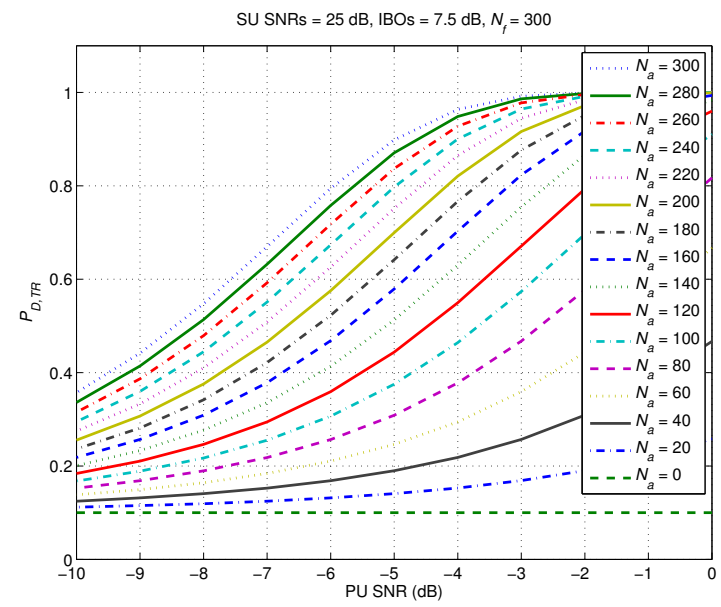

Fig. 9. Transient phase detection performance. The case with increased signal band power dynamics and nonlinear PA operation.

the overall sensing period. Also here, the detector is designed to achieve false alarm probability of $P_{F A}=0.1$ with $N_{a}=0$, and perfect knowledge of the effective noise variance $\bar{\sigma}^{2}$ is assumed. Comparing the performance curves in Fig. 8 with those of Fig. 9, the reduction in the effective PU SNR due to the increased spectral regrowth becomes evident. In order to keep up with the performance achieved with linear PA (or with high back-off values), the integration time should be increased accordingly.

\section{CONCLUDING REMARKS}

In this paper, we have presented a silent sensing subband based spectrum monitoring technique to be applied in data-receiving filter bank multicarrier secondary devices. An expression was derived for the test statistic of the energy detector, under so-called transient phase where reappearance of the PU falls arbitrarily within the integration period. This analytical model was verified by experimental data harvested from an FBMC/OQAM testbed. Moreover, the sensitivity of the proposed scheme to nonlinear power amplifier induced spectral regrowth was analyzed.
Throughout the paper, we assumed perfect knowledge of the effective noise variance in the monitoring receiver. In practice, an estimate of this variance has to be provided for the detector to enable proper threshold setting. The following approach could be taken to facilitate the required estimation. A spectral regrowth analysis for a specific PA could be carried out offline and results tabulated for different back-offs as in Table I. The relative level of the expected regrowth component, corresponding to the back-off applied in the transmitter, could then be signalled to the receiver side in a form of a few-bit side information within the secondary data. The secondary receivers, which are not actively decoding the ongoing secondary data but only monitor the dedicated sensing subbands, could measure the monitoring subband to obtain an estimate of the noise variance. Recall that, a careful pre-transmission sensing has been carried out prior establishing the secondary link.

It should also be noted that the sensing subbands enable an efficient and fast distribution of the reappeared $P U$ detected message within the SU system. The idea here is to apply the boosting protocol proposed in [15] and to beacon a high-power pilot-like signal over the monitoring subbands to alarm SUs for quick channel vacation.

\section{REFERENCES}

[1] Q. Zhao and B. M. Sadler, "A survey of dynamic spectrum access: signal processing, networking, and regulatory policy," IEEE Signal Proc. Mag., vol. 24, pp. 79-89, May. 2007.

[2] J. Mitola and G. Q. Maguire, "Cognitive radio: making software radios more personal," IEEE Pers. Commun. Mag., vol. 6, pp. 13-18, Aug. 1999.

[3] S. Haykin, "Cognitive radio: brain-empowered wireless communications," IEEE J. Select. Areas Commun, vol. 23, pp. 201-220, Feb. 2005.

[4] M. Höyhtyä, A. Hekkala, M. Katz, and A. M. Mämmelä, "Spectrum awareness: techniques and challenges for active spectrum sensing," Cognitive Wireless Networks, F. H. P. Fitzek and M. D. Katz, Eds. Dordrecht, Netherlands: Springer, 2007, ch. 18, pp. 353-372.

[5] C. Cordeiro, M. Ghosh, D. Cavalcanti, and K. Chappali, "Spectrum sensing for dynamic spectrum access of TV bands," in Proc. 2nd Int. Conf. Cognitive Radio Oriented Wireless Networks and Communications, Orlando, Florida, USA, Aug. 2007, pp. 225-233.

[6] B. Farhang-Boroujeny, "Filter bank spectrum sensing for cognitive radios," IEEE Trans. on Signal Proc., vol. 56, pp. 1801-1811, May 2008.

[7] H. Zhang, D. Le Ruyet, and M. Terre, "Spectral efficiency comparison between OFDM/OQAM and OFDM based CR networks," Wireless Communications and Mobile Computing, Wiley InterScience, vol. 9, pp. 1487 1501, Nov. 2009.

[8] European Union's 7th Framework Programme: Project PHYDYAS, website: www.ict-phydyas.org.

[9] P. Siohan, C. Siclet, and N. Lacaille, "Analysis and design of OFDM/OQAM systems based on filter bank theory," IEEE Trans. on Signal Proc., vol. 50, pp. 1170-1183, May 2002.

[10] S. Mirabbasi and K. Martin, "Overlapped complex-modulated transmultiplexer filters with simplified design and superior stopbands," IEEE Trans. Circuits Syst. II, vol. 50, pp. 456-469, Aug. 2003.

[11] R. Tandra and A. Sahai, "SNR walls for signal detection," IEEE J. Select. Topics Signal Proc., vol. 2, pp. 4-17, Feb. 2008.

[12] "INFSO-ICT-211887 Project PHYDYAS, Deliverable 8.2: Space-time spectrum sensing, algorithms and software description," Feb. 2010.

[13] "INFSO-ICT-211887 Project PHYDYAS, Deliverable 8.1: Application of the FBMC physical layer in a cognitive radio scenario," July 2009.

[14] C. Rapp, "Effects of HPA-nonlinearity on a 4-DPSK/OFDM-signal for a digital sound broadcasting system," in Proc. 2nd European Conf. Satellite Communications, Liege, Belgium, Oct. 1991, pp. 179-184.

[15] T. Weiss, J. Hillenbrand, A. Krohn, and F. K. Jondral "Efficient signaling of spectral resources in spectrum pooling systems," in Proc. 10th Symp. Communications and Vehicular Technology, Eindhoven, Netherlands, Nov. 2003. 